\title{
Modulation of osteoclastogenesis by antihypertensive drugs
}

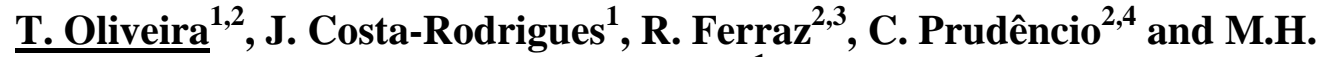 \\ Fernandes ${ }^{1}$ \\ ${ }^{1}$ Laboratory for Bone Metabolism and Regeneration, Faculty of Dental Medicine, University of \\ Porto, Portugal. \\ ${ }^{2}$ CISA/CQB Escola Superior de Tecnologia da Saúde do Porto Instituto Politécnico do Porto, \\ Portugal. \\ ${ }^{3}$ REQUIMTE-CQFB, Faculdade de Ciências e Tecnologia da Universidade Nova de Lisboa, \\ Portugal. \\ ${ }^{4}$ Centro de Farmacologia e Biopatologia Química (U38-FCT), Faculty of Medicine, University \\ of Porto, Portugal.
}

Despite its rigid structure, bone is a dynamic tissue that is in constant remodeling. This process requires the action of the bone-resorbing osteoclasts and the bone-synthesing osteoblasts. One of the adverse effects attributed to some antihypertensive agents is the ability to alter normal bone metabolism. However, their effective actions on human bone cells remain to be clarified. In this work, the effects of five calcium channel blockers, a class of antihypertensive drugs (AHDs), were investigated on osteoclastic differentiation.

Osteoclastic cell cultures were established from precursor cells isolated from human peripheral blood, and were maintained in the absence (control) or in the presence of $10^{-}$ ${ }^{8}-10^{-4} \mathrm{M}$ of different AHDs (amlodipine, felodipine, diltiazem, lercanidipine and nifedipine). Cell cultures were characterized throughout a 21 day period for tartrateresistant acid phosphatase (TRAP) activity, number of TRAP+ multinucleated cells, presence of cells with actin rings and expressing vitronectin and calcitonin receptors, and apoptosis rate. Also, the involvement of several signaling pathways on the cellular response was addressed.

It was observed that the tested AHDs had the ability to differentially affect osteoclastogenesis. At low doses, amlodipine and felodipine caused an increase on osteoclastic differentiation, while the other drugs inhibited it. At higher doses, all the molecules caused a decrease on the process. The tested AHDs also showed different effects on the analysed signaling pathways.

In conclusion, AHDs appeared to have a direct effect on human osteoclast precursor cells, affecting their differentiation. Interestingly, some of them increased while others inhibited the process. Unraveling the mechanisms beneath these observations might help to explain the adverse effects on bone tissue described for this drug class. 